\title{
LA-UR-20-26250
}

Approved for public release; distribution is unlimited.

Title: Data-Parallel Programming in PyTorch for Volumetric Radiation Transport Solvers

Author(s): $\quad$ Marshak, Nathan Xu

Grosset, Andre Vincent Pascal

Ahrens, James Paul

Intended for: Presentation

Issued: 
Disclaimer:

Los Alamos National Laboratory, an affirmative action/equal opportunity employer, is operated by Triad National Security, LLC for the National Nuclear Security Administration of U.S. Department of Energy under contract 89233218CNA000001. By approving this article, the publisher recognizes that the U.S. Government retains nonexclusive, royalty-free license to publish or reproduce the published form of this contribution, or to allow others to do so, for U.S. Government purposes. Los Alamos National Laboratory requests that the publisher identify this article as work performed under the auspices of the U.S. Department of Energy. Los Alamos National Laboratory strongly supports academic freedom and a researcher's right to publish; as an institution, however, the Laboratory does not endorse the viewpoint of a publication or guarantee its technical correctness. 


\section{Data-Parallel Programming in}

PyTorch for Volumetric

Radiation Transport Solvers

Nathan Marshak (LANL intern)

Hannah Ross (LBNL Domain Scientist)

Pascal Grosset \& Jim Ahrens (LANL Mentors)

Data Science at Scale Summer School Staff (LANL) 


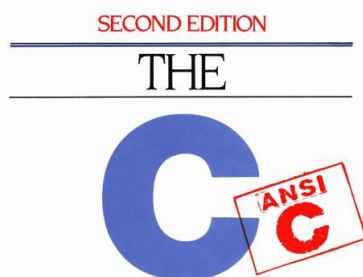

PROGRAMMING LANGUAGE

BRIAN W. KERNIGHAN

RITCHIE

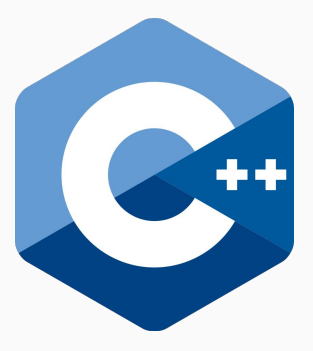

(2) NVIDIA. CUDA.
High performance

Labor intensive 


\section{¿ PyTorch}

High-level interface to the GPU

Good distributed capability

Ridiculously popular

Backed by people with $\$ \$ \$$

For ML, not scientific computing

No low-level GPU programming 


\section{Data-parallel primitives}

- Add

- SAXPY

- Scan

- Reduce

- Sort

- Rotate

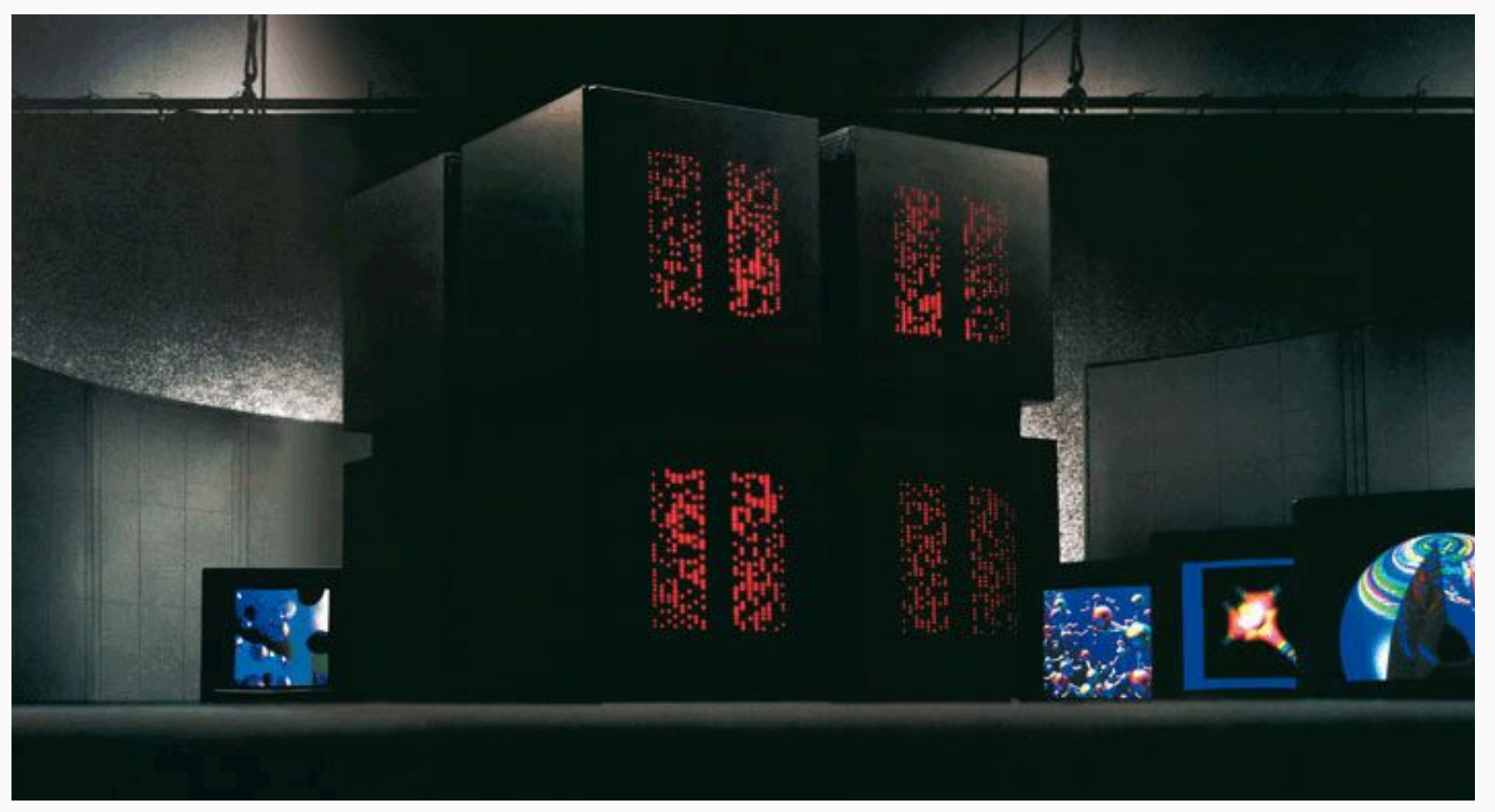


Can we use data-parallel primitives in PyTorch for radiation transport?

1. How efficient are data-parallel primitives in PyTorch?

2. Can we implement a volume renderer with them in PyTorch?

3. How do we solve a scientific problem? 


\section{How efficient are data-parallel primitives in PyTorch?}

Time for prefix-sum

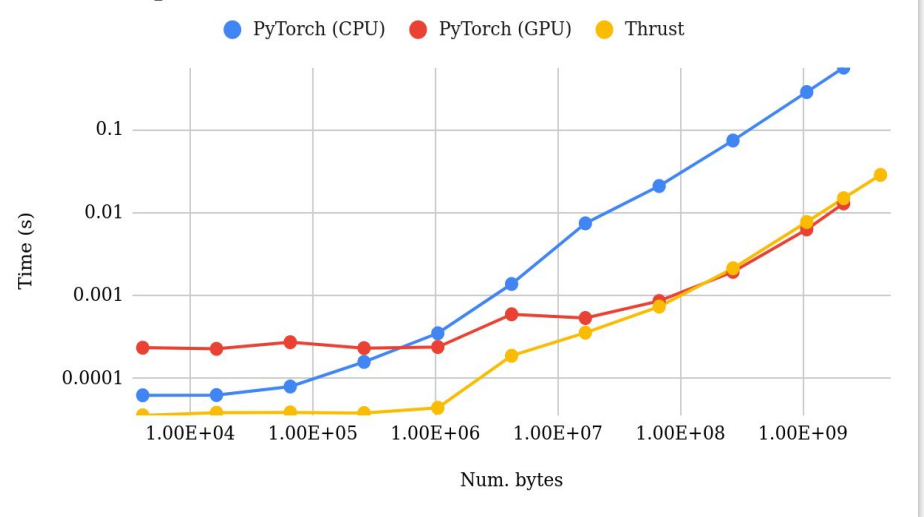

Time for 50 repeated SAXPYs

PyTorch (CPU) PyTorch (GPU) Thrust

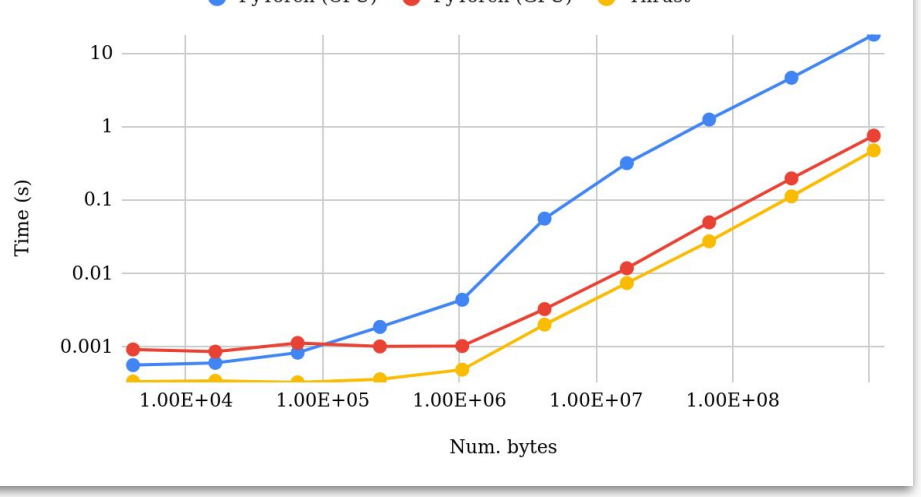

Num. image elements vs. time to rotate image - PyTorch (CPU) PyTorch (GPU) OpenCV+CUDA

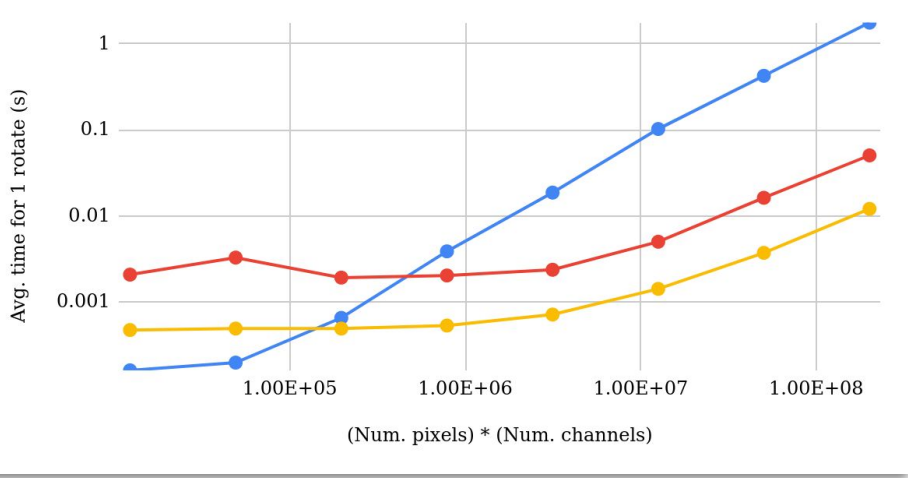

Time for sort

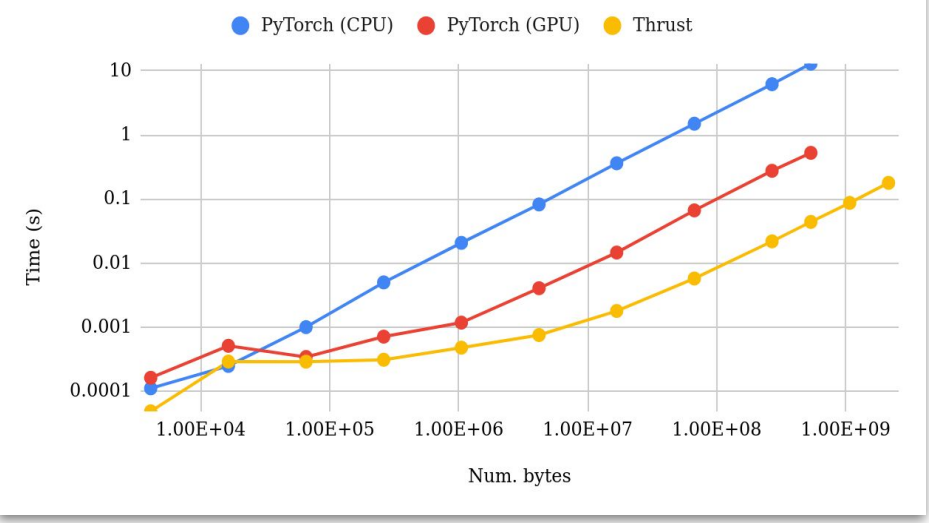




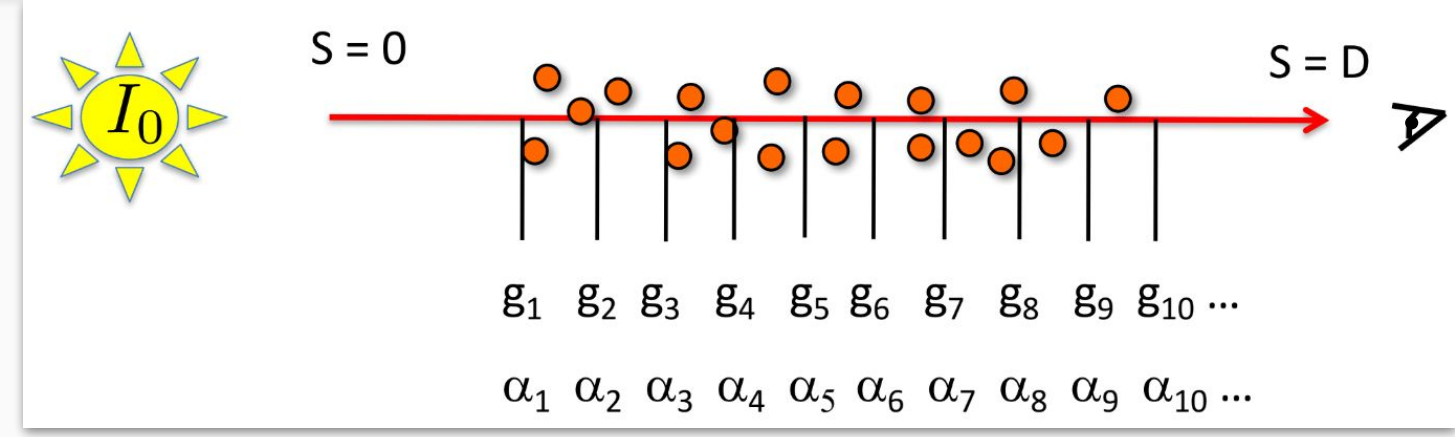

Analytic RTE solution: $\quad I(D)=I_{0} e^{-\int_{0}^{D} \kappa(t) \mathrm{d} t}+\int_{0}^{D} g(s) e^{-\int_{s}^{D} \kappa(t) \mathrm{d} t} \mathrm{~d} s$

$$
\text { implementation }\left\{\begin{array}{l}
C=C_{-} \theta \\
T=1-\alpha[0] \\
\text { for } i=1 \rightarrow N: \\
C=g[i]+(1-\alpha[i]) * C \\
\text { end }
\end{array}\right.
$$




$$
\begin{aligned}
& I(D)=I_{0} e^{-\int_{0}^{D} \kappa(t) d t}+\int_{0}^{D} g(s) e^{-\int_{s}^{D} \kappa(t) d t} d s \\
& \approx I_{0} \prod_{i=1}^{N}\left(1-\alpha_{i}\right)+\sum_{i=1}^{N} g_{i} \prod_{j=i+1}^{N}\left(1-\alpha_{j}\right)
\end{aligned}
$$

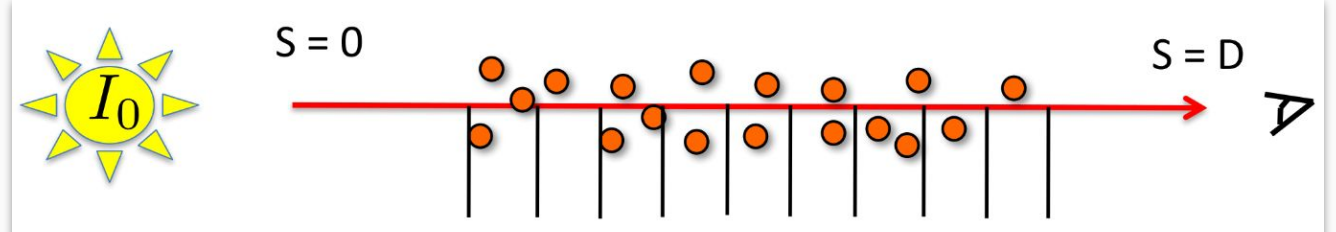

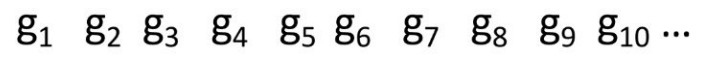

$$
\begin{aligned}
& \begin{array}{lllllllllllllllllllllll}
\alpha_{1} & \alpha_{2} & \alpha_{3} & \alpha_{4} & \alpha_{5} & \alpha_{6} & \alpha_{7} & \alpha_{8} & \alpha_{9} & \alpha_{10} & \ldots
\end{array}
\end{aligned}
$$




\section{Volume rendering with data-parallel primitives}

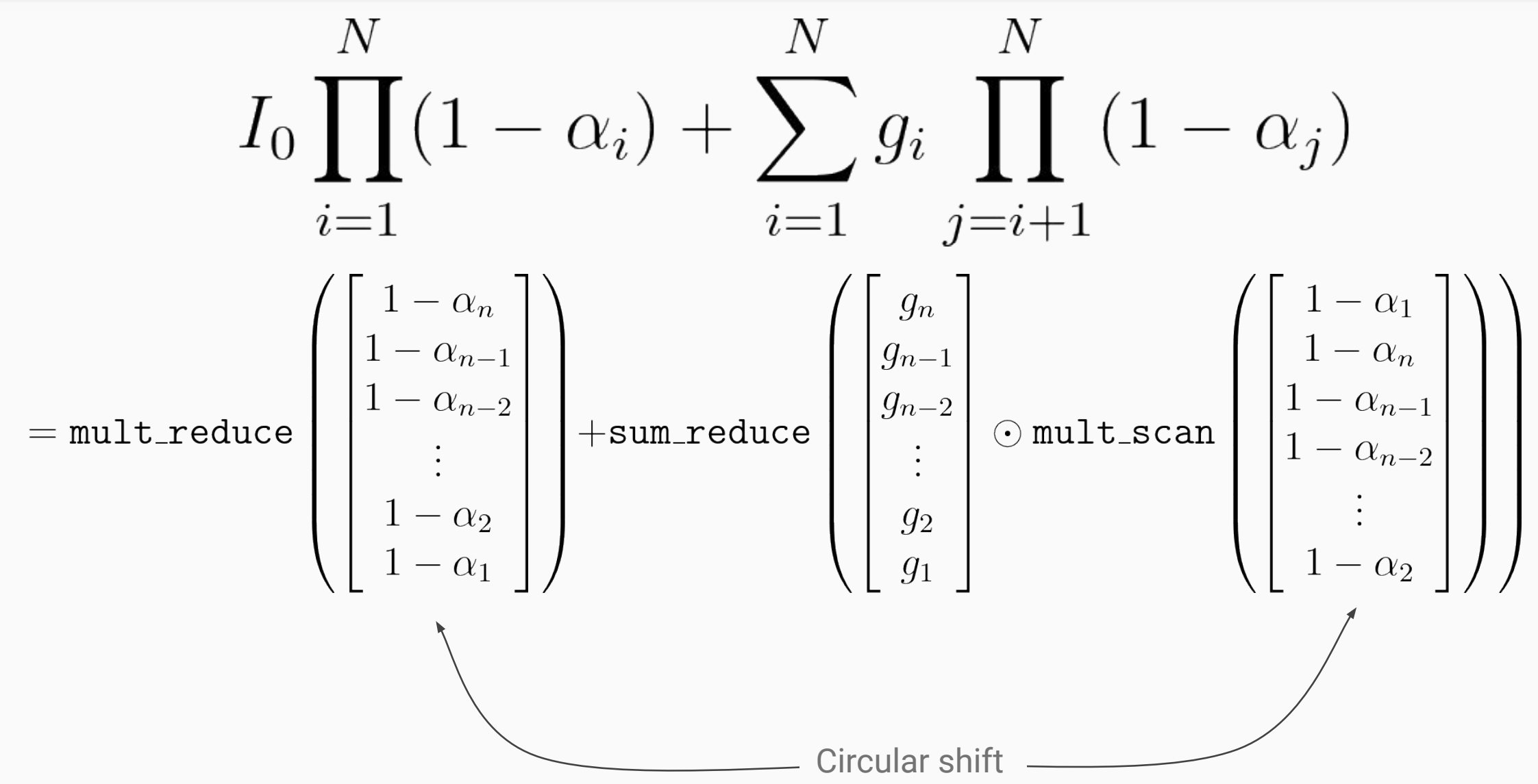


Ours vs. OpenVKL vs. ParaView

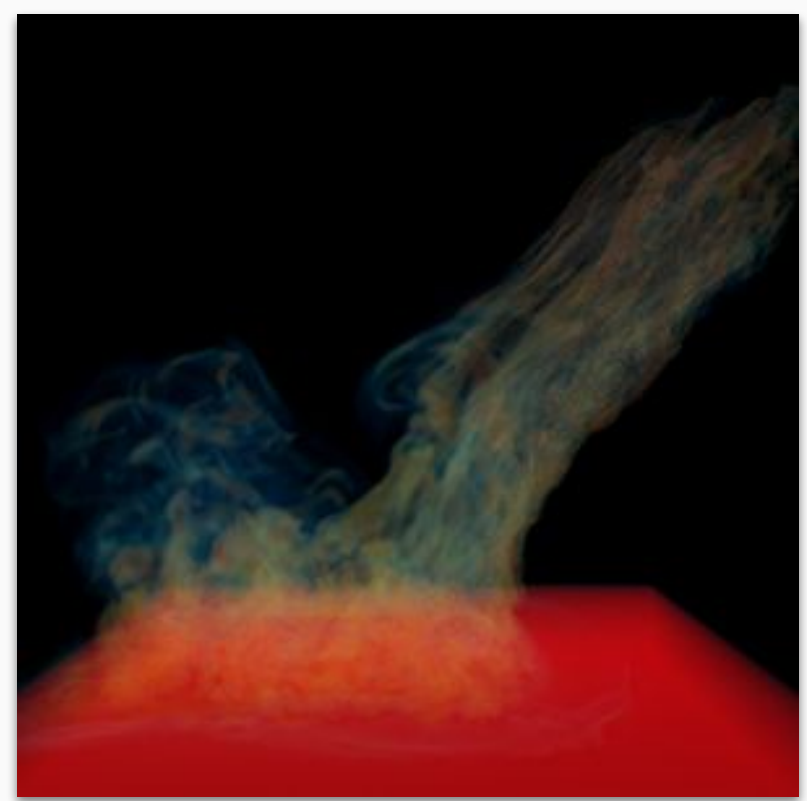

Ours

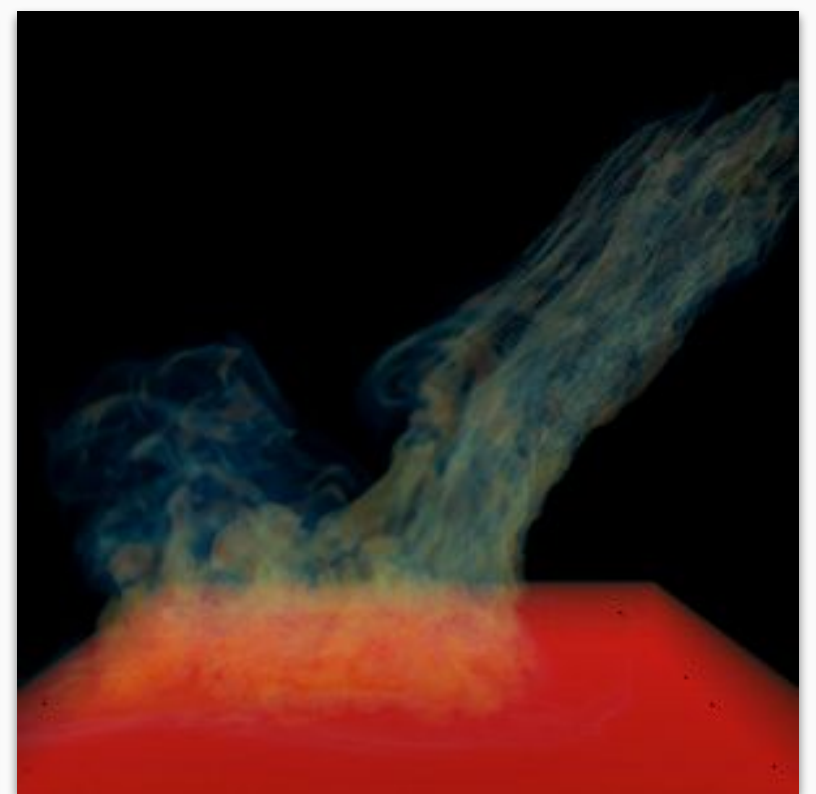

OpenVKL

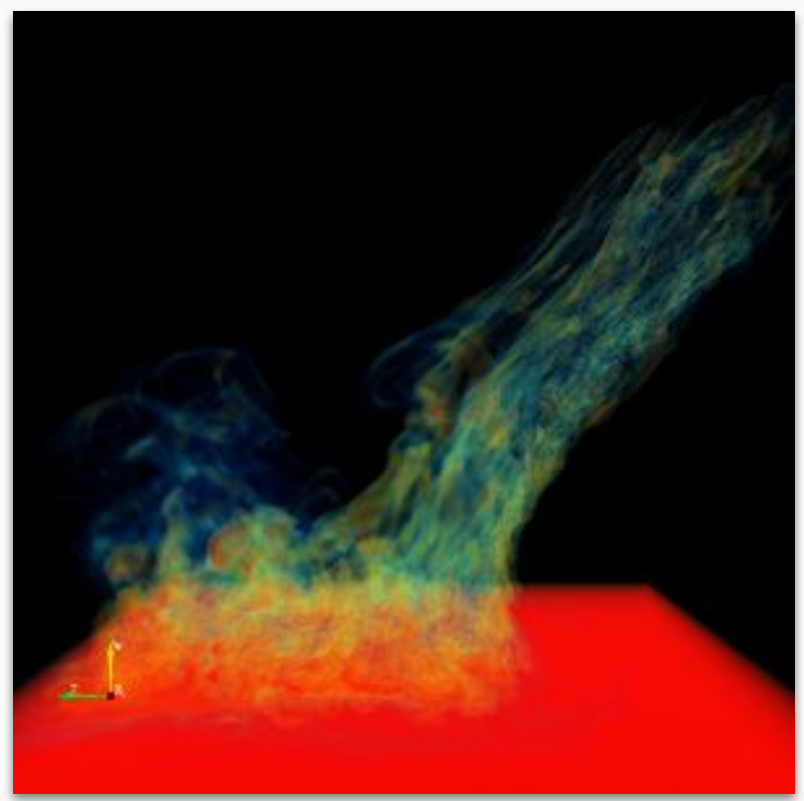

ParaView 


\begin{tabular}{|l|l|l|}
\hline Renderer & $\begin{array}{l}\text { Frames / sec. } \\
\text { (Higher is better) }\end{array}$ & $\begin{array}{l}\text { Sec. / frame } \\
\text { (Lower is better) }\end{array}$ \\
\hline Ours, orthographic (GPU) & 17.7 & 0.0564 \\
\hline Ours, perspective (GPU) & 4.76 & 0.210 \\
\hline ParaView (GPU) & 67.61 & 0.0148 \\
\hline OpenVKL (CPU) & 19.2 & 0.052 \\
\hline
\end{tabular}




\section{Volume rendering integral \& photoionization rate equation}

$\Delta \frac{\Delta}{I_{0}}$

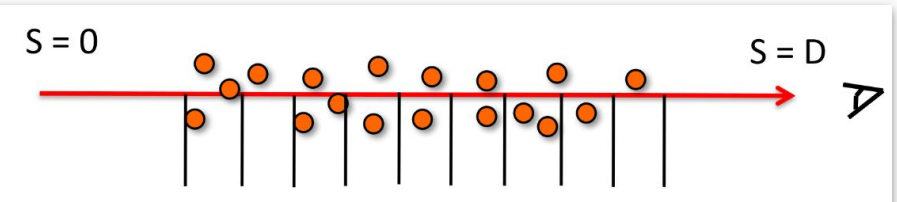

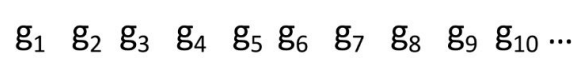

$\begin{array}{lllllllllllllll}\alpha_{1} & \alpha_{2} & \alpha_{3} & \alpha_{4} & \alpha_{5} & \alpha_{6} & \alpha_{7} & \alpha_{8} & \alpha_{9} & \alpha_{10} & \ldots\end{array}$

$I(D)=I_{0} e^{-\int_{0}^{D} \kappa(t) \mathrm{d} t}+\int_{0}^{D} g(s) e^{-\int_{s}^{D} \kappa(t) \mathrm{d} t} \mathrm{~d} s$

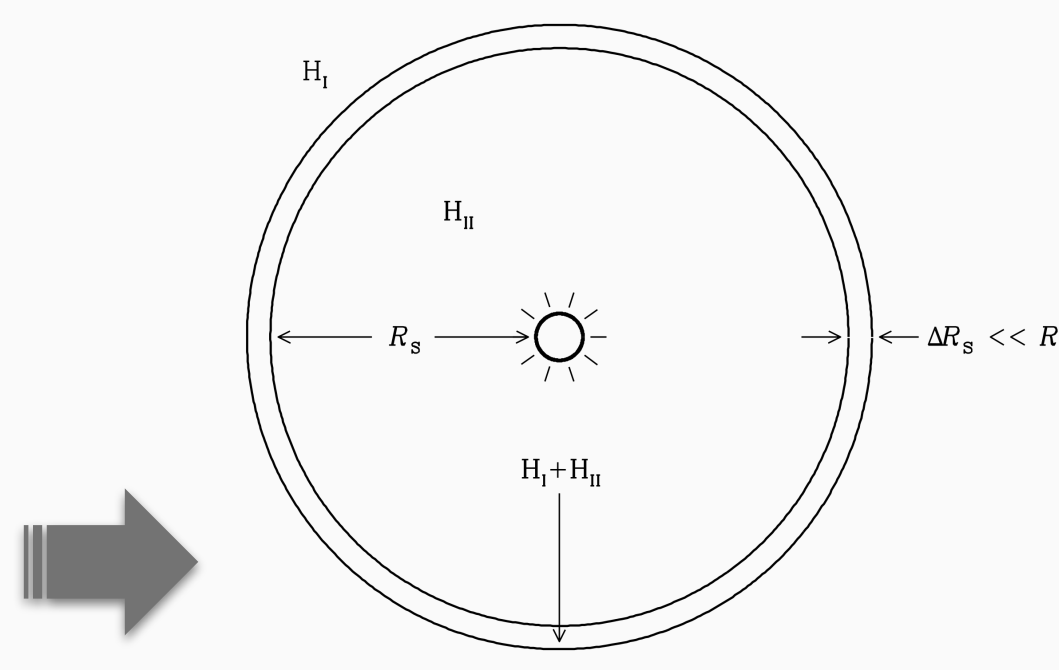

$$
\Gamma_{\text {local }}(r)=\frac{1}{4 \pi r^{2}} \int_{v_{\text {th }}}^{\infty} \frac{L_{v} \sigma_{v} \mathrm{e}^{-\tau_{v}(r)}}{h v} \mathrm{~d} v
$$




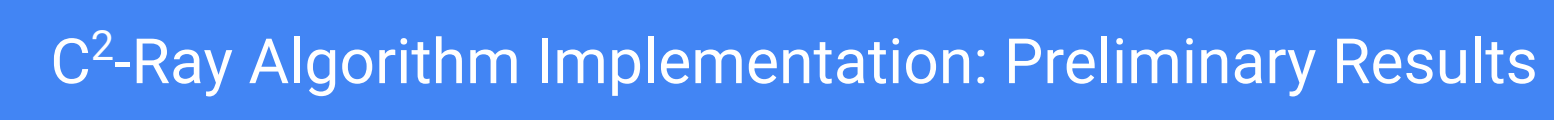

Current cell: 0 , iteration \# 0

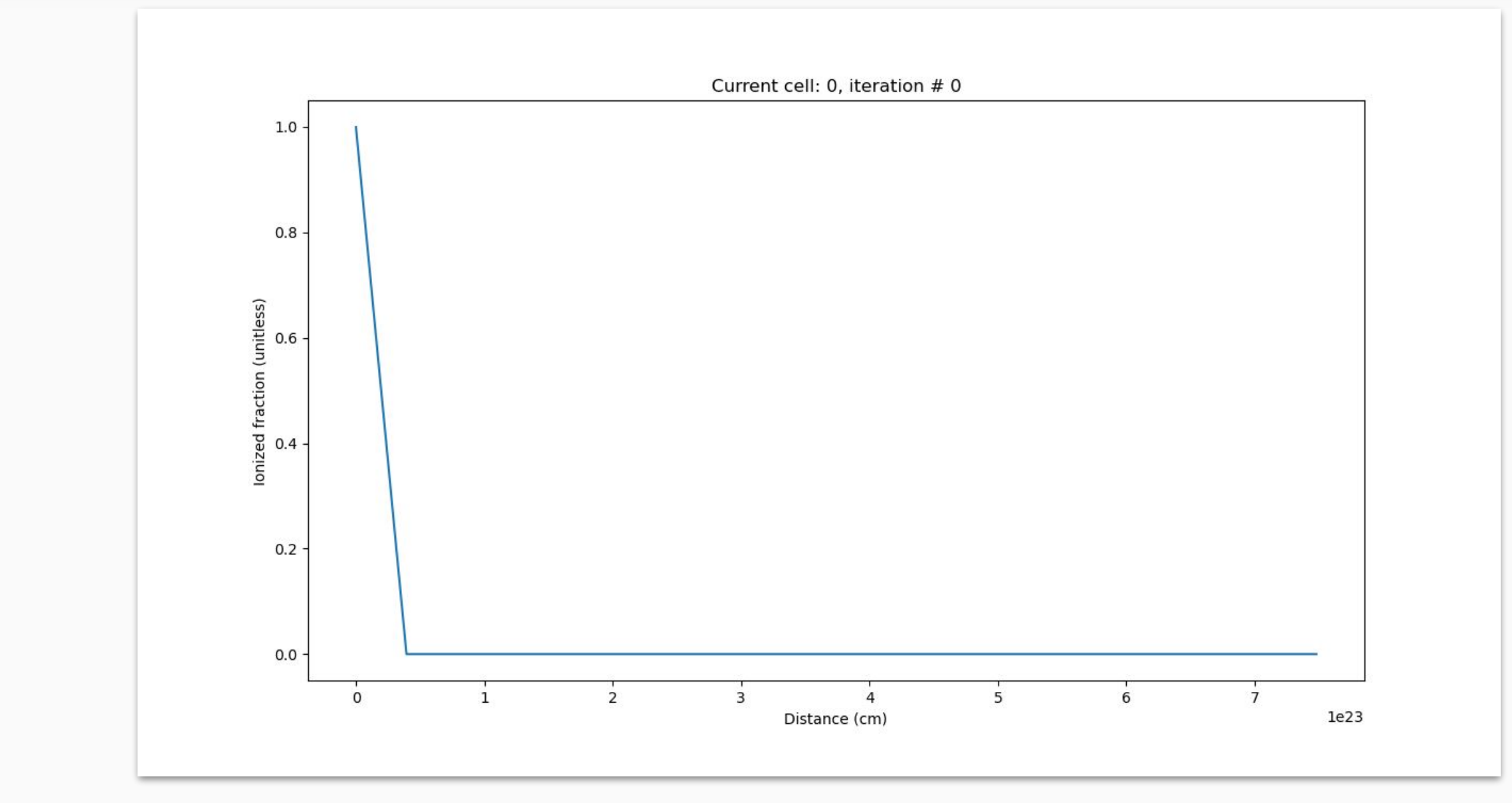




\section{Conclusion}

1. How efficient are data-parallel primitives in PyTorch?

2. Can we implement a volume renderer with them in PyTorch?

3. How do we solve a scientific problem?
1. Data-parallel primitives are reasonably fast in PyTorch, although rotation and sort are suboptimal.

2. We absolutely can implement reasonably efficient volume renderer in PyTorch.

a. It is not quite as fast as a native GPU renderer due to rotation being slower than native CUDA code.

3. We're learning how to solve a scientific problem

a. We found a well-defined theoretical relationship between volume rendering and what the $\mathrm{C}^{2}$-Ray code is doing.

b. We have encouraging initial test results, despite running into a convergence problem. 


\section{Sources cited}

1. Badal, Andreu, et al. "penMesh-Monte Carlo radiation transport simulation in a triangle mesh geometry." IEEE Transactions on Medical Imaging 28.12 (2009): 1894-1901.

2. Blelloch, Guy E. Vector models for data-parallel computing. Vol. 2. Cambridge: MIT press, 1990.

3. Haider, Jibran. An Upwind Cell Centred Finite Volume Method for Large Strain Explicit Solid Dynamics in OpenFOAM. Diss. Swansea University, 2018.

4. Howell, John R., M. Pinar Menguc, and Robert Siegel. Thermal radiation heat transfer. CRC press, 2010.

5. Maeda, Tomohiro, et al. "Thermal non-line-of-sight imaging." 2019 IEEE International Conference on Computational Photography (ICCP). IEEE, 2019.

6. Mellema, Garrelt, et al. "C2-ray: A new method for photon-conserving transport of ionizing radiation." New Astronomy 11.5 (2006): 374-395.

7. Morrical, Nate, et al. "Efficient space skipping and adaptive sampling of unstructured volumes using hardware accelerated ray tracing." 2019 IEEE Visualization Conference (VIS). IEEE, 2019.

8. Villefranque, Najda, et al. "A Path-Tracing Monte Carlo Library for 3-D Radiative Transfer in Highly Resolved Cloudy Atmospheres." Journal of Advances in Modeling Earth Systems 11.8 (2019): 2449-2473.

9. Woodcock, E., et al. "Techniques used in the GEM code for Monte Carlo neutronics calculations in reactors and other systems of complex geometry." Proc. Conf. Applications of Computing Methods to Reactor Problems. Vol. 557. No. 2. 1965. 


\section{Convergence and optical thickness}

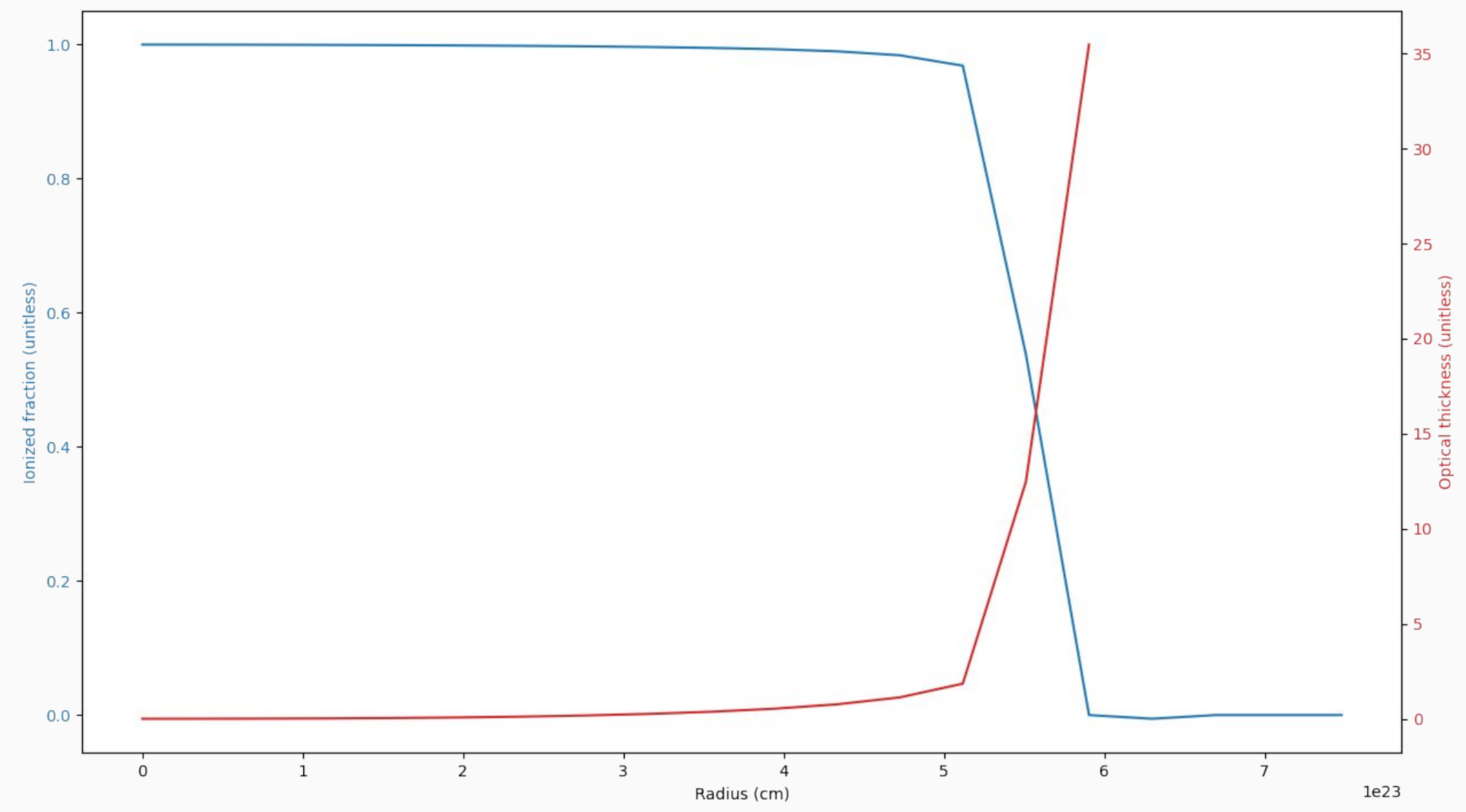




\section{Linear interpolation in pytorch}

7. veeresh_d

$\mathrm{Hi}$ all.

I am newbei to the pytorch. is there any interpolation (linear) function that is similar to the np.interp 21 function.

Please let me know if you need any details? Thanks in advance.

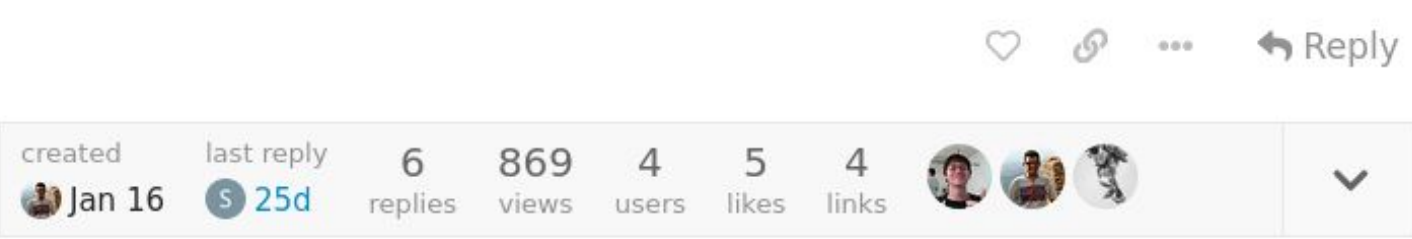

$\mathrm{Hi}$,

I'm afraid there is none.

If you're fine with a non-differentiable, cpu-only implementation, you can use the numpy one as a workaround: $r e s=\operatorname{torch}$. from_numpy (np.interp (x.numpy (), fx.numpy (), fp. numpy ()). 


\section{MONTHS LATER}

I just wrote a package that implements this (GPU-compatible \& differentiable).

https://github.com/sbarratt/torch_interpolations 18

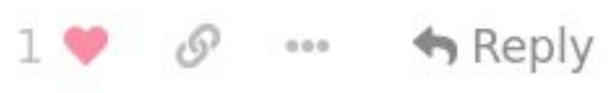


How efficient are data-parallel primitives in PyTorch?

Time for sort, scan, add, rotate

Scan Time Sort Time Avg. Single SAXPY Time

Avg. single rotate time

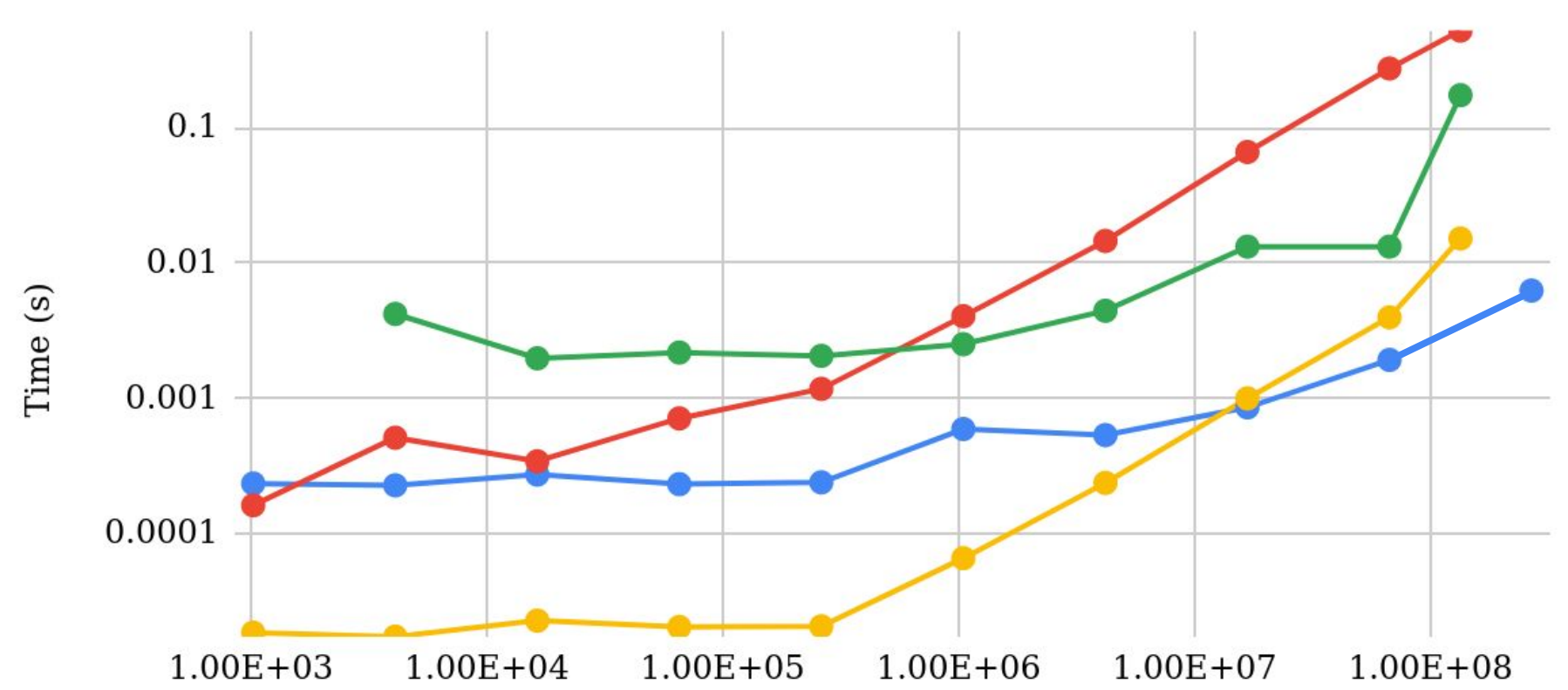

Num. array elements 


\section{Scan Benchmark}

\section{Time for prefix-sum}

PyTorch (CPU) PyTorch (GPU) Thrust

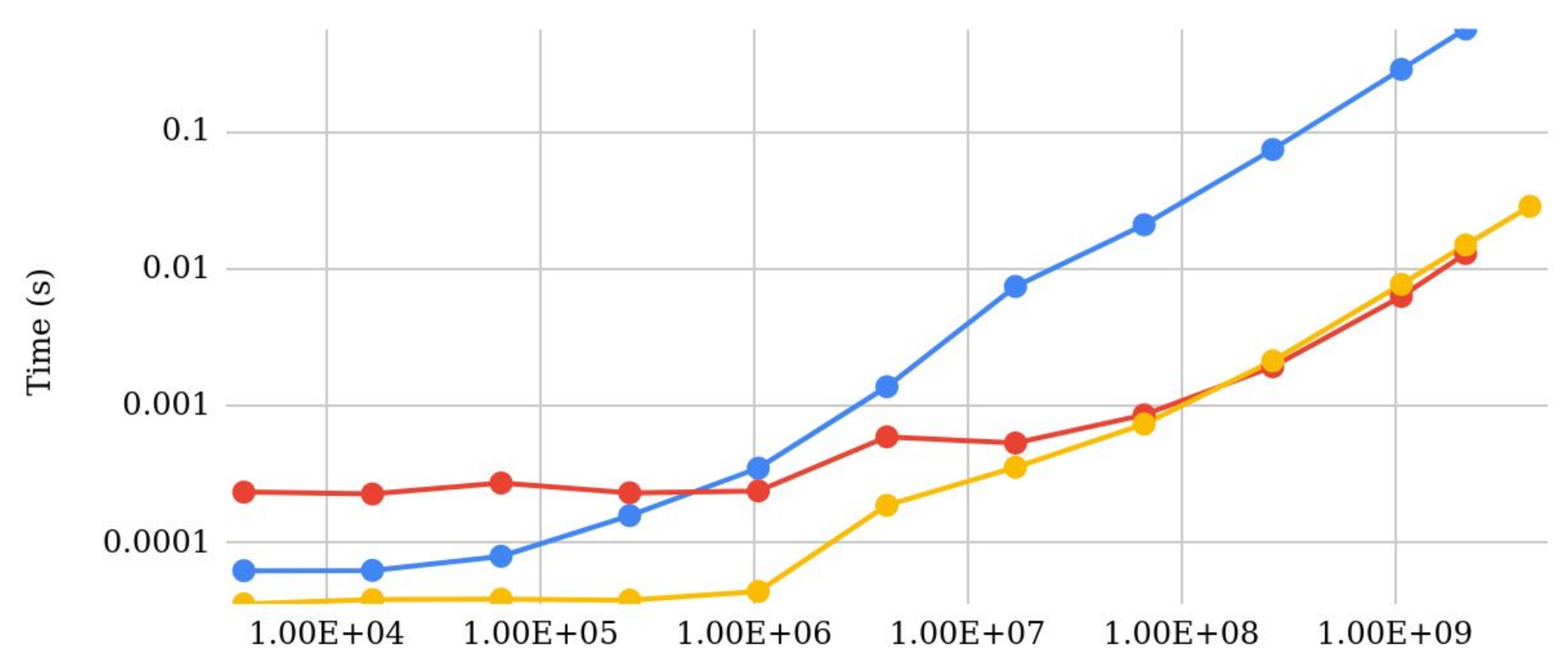

Num. bytes 


\section{SAXPY Benchmark}

\section{Time for 50 repeated SAXPYs}

PyTorch (CPU)

PyTorch (GPU)

Thrust

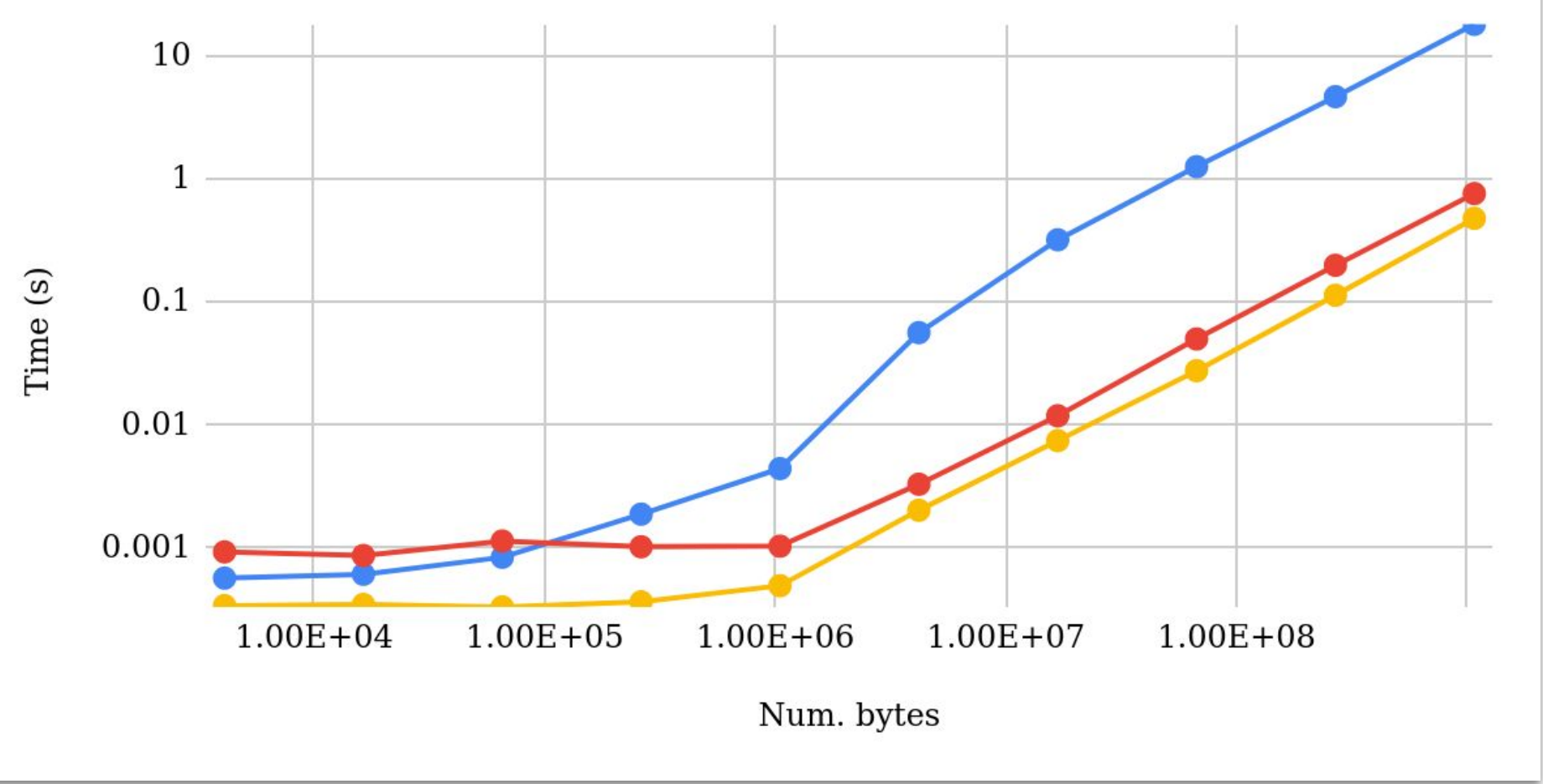




\section{Sort Benchmark}

\section{Time for sort}

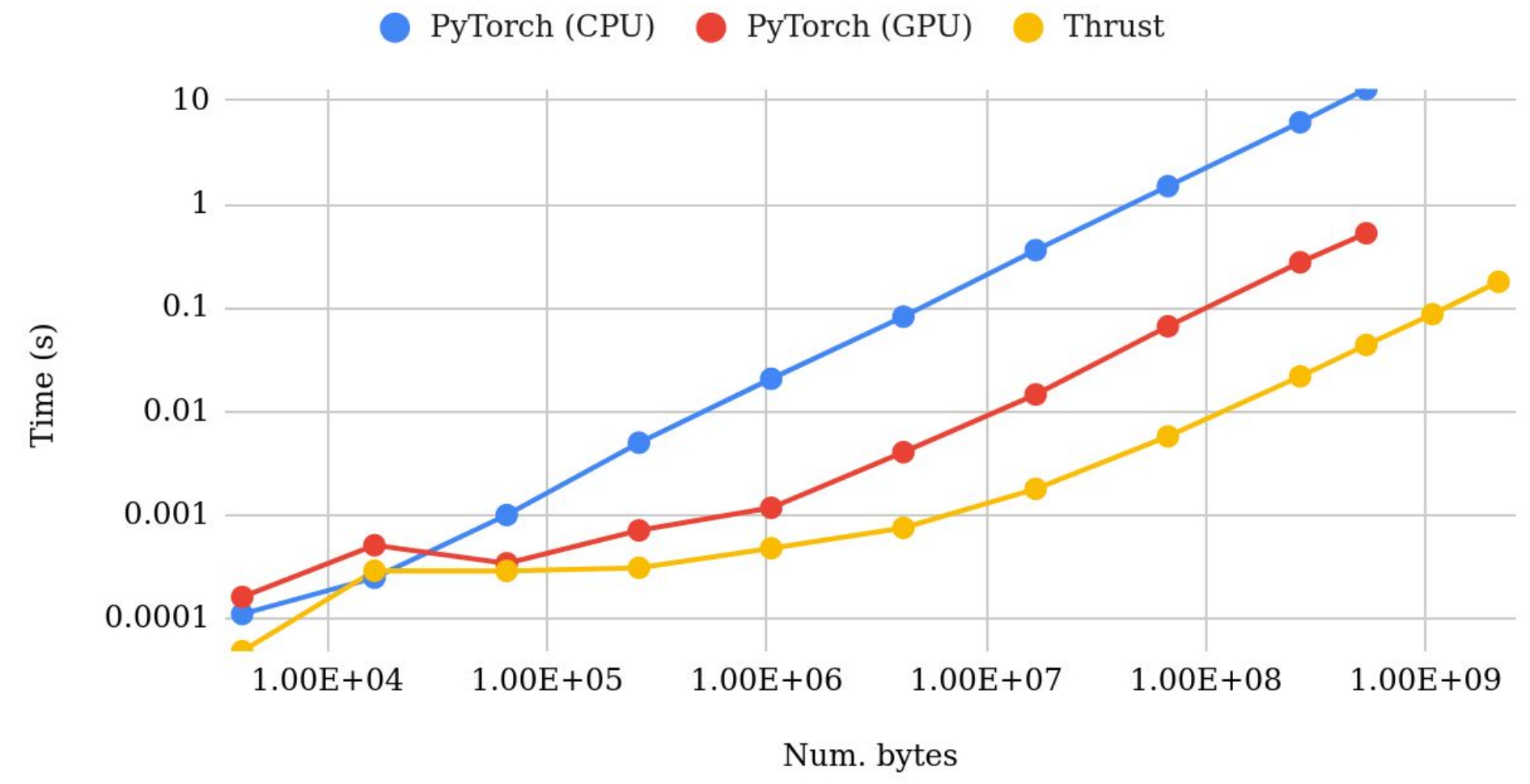




\section{Segmented reduce benchmark}

Time for reduce (8 segments)

PyTorch (CPU) PyTorch (GPU) Thrust

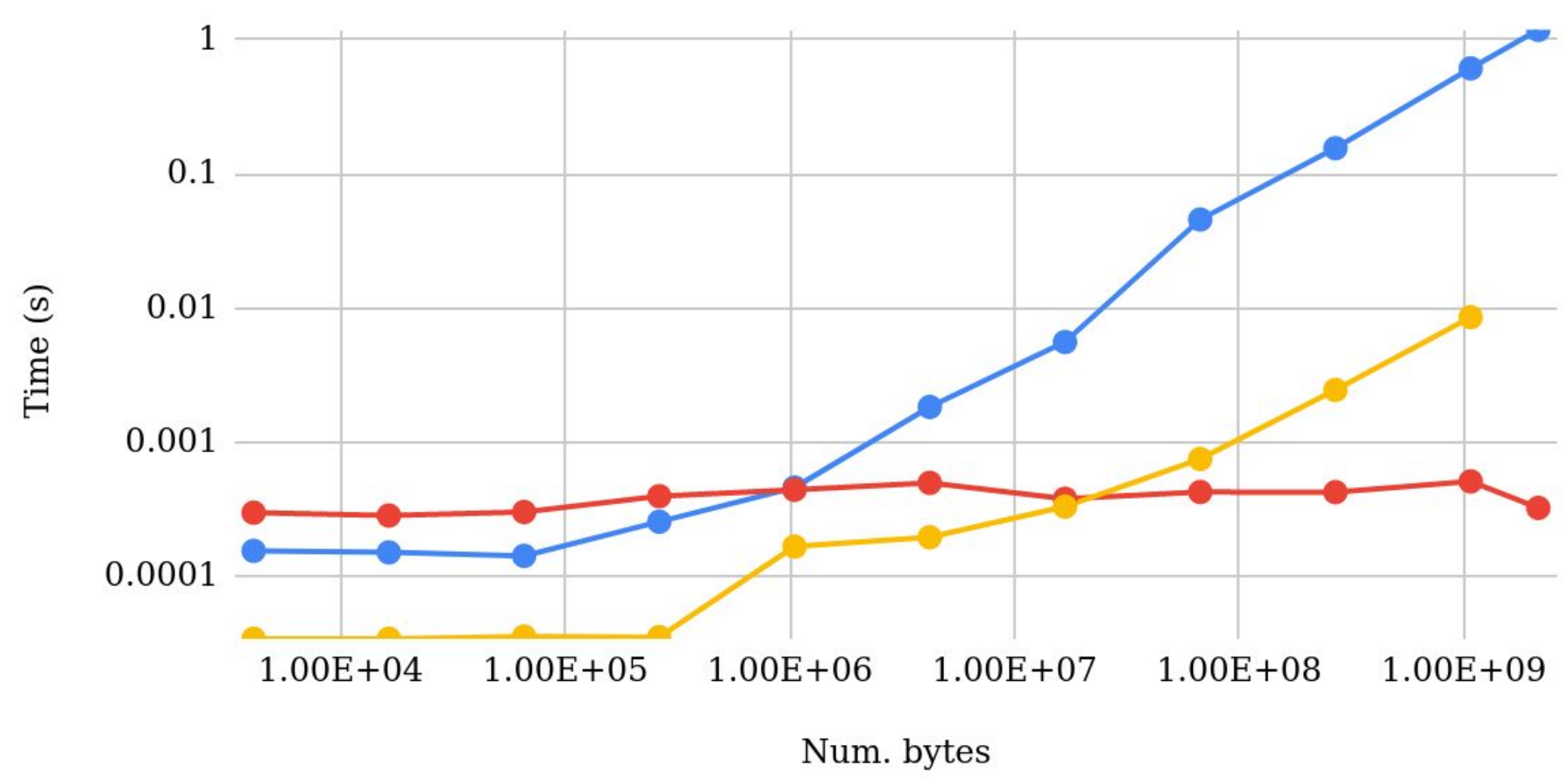




\section{Image Rotation Benchmark}

Num. image elements vs. time to rotate image

- PyTorch (CPU) PyTorch (GPU) OpenCV+CUDA

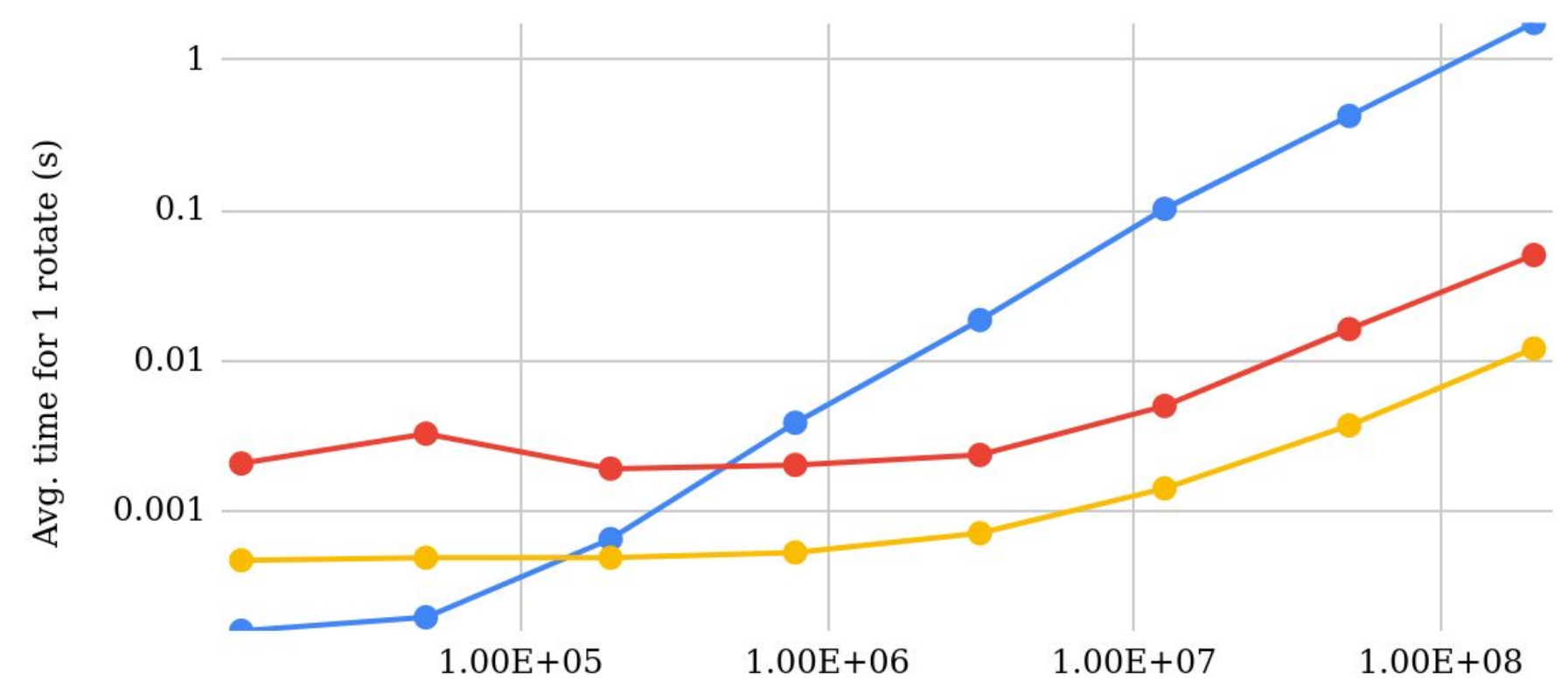

(Num. pixels) * (Num. channels) 


\section{Ratio of scan, sort, rot time to SAXPY time}

(Scan Time)/(Avg. single SAXPY time) (Sort Time)/(Avg. single SAXPY time)

(Avg. single SAXPY time)/(Avg. single SAXPY time)

(Avg. single rot. time)/(Avg. single SAXPY time)

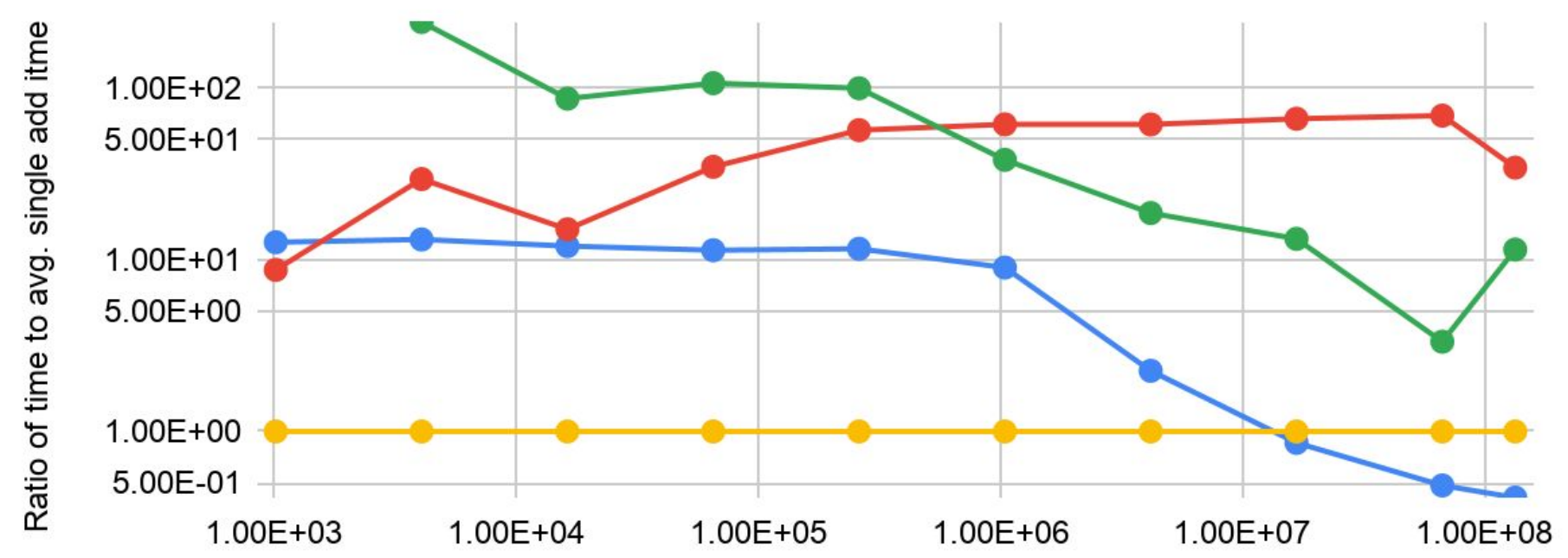

Num. array elements 


\section{Profiler output}

pyscirt.py:63(rot_and_composite_ortho) $\overline{0} .56 \overline{4} \mathrm{~s}$

functional.py:3235(affine_grid)

$$
0.221 \mathrm{~s}
$$

:0(<built-in method affine_grid_generator >) $0.221 \mathrm{~s}$ functional.py:3120(grid_sample) $0.198 \mathrm{~s}$

$\sim: 0$ (<built-in method grid_sampler >) $0.198 \mathrm{~s}$ pyscirt.py:41(reduce_composite) $0.138 \mathrm{~s}$

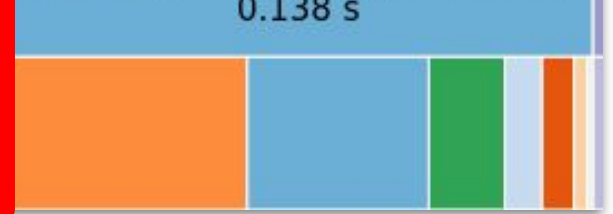


Volume rendering with data-parallel primitives
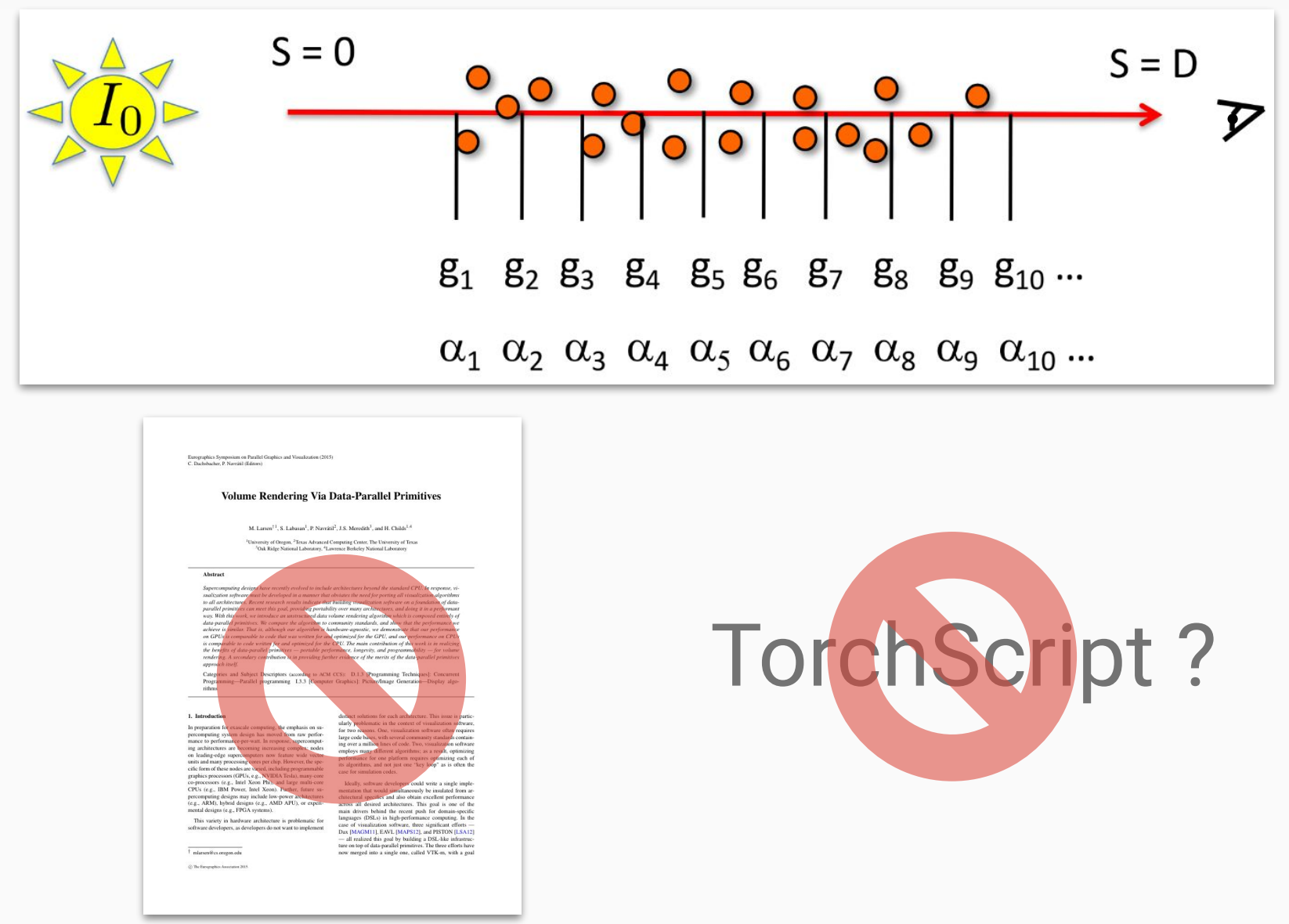

TorchScript? 